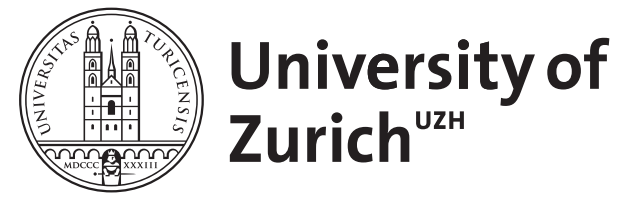

\title{
Globale distributive Gerechtigkeit
}

\author{
Marti, Urs
}

Posted at the Zurich Open Repository and Archive, University of Zurich ZORA URL: https://doi.org/10.5167/uzh-80821

Book Section

Published Version

Originally published at:

Marti, Urs (2003). Globale distributive Gerechtigkeit. In: Marti, Urs; Kohler, Georg. Konturen der neuen Welt(un)ordnung : Beiträge zu einer Theorie der normativen Prinzipien internationaler Politik. Berlin/New York: de Gruyter, 345-361. 


\title{
Globale distributive Gerechtigkeit
}

\author{
Urs Marti
}

Die weltweit ungleiche Verteilung der Bedingungen für ein Leben in Freiheit, Sicherheit und Wohlstand ist offensichtlich, umstritten ist, ob es sich dabei um eine von Menschen zu verantwortende Ungerechtigkeit handelt. Eine unparteiische Antwort auf die Frage fällt naturgemäß nicht leicht; wer mit seinem Anteil zufrieden ist, neigt dazu, im Geschick der anderen eher ein Unglück denn eine Ungerechtigkeit zu sehen. Die politische Philosophie müsste folglich ein Sensorium für Ungerechtigkeit entwickeln, ehe sie Gerechtigkeitsprinzipien begründet (vgl. Shklar, 1992). Das ist ihr zwar allenfalls beschränkt möglich, dennoch lohnt es sich, zu prüfen, in welchem Maß Gerechtigkeitstheoretikerinnen und -theoretiker über ein entsprechendes Sensorium verfügen. Wie ein Blick auf die einschlägige Literatur zeigt, bestehen diesbezüglich markante Unterschiede.

Ich werde zunächst kurz die Debatte zwischen Anhängern und Gegnern kosmopolitischer Gerechtigkeitstheorien nachzeichnen. Anhand eines knappen Überblicks über internationale wirtschaftspolitische Kontroversen der Nachkriegszeit werde ich anschließend an eine Erfahrung von Ungerechtigkeit erinnern, die in der philosophischen Debatte meist verdrängt wird. Es folgen Überlegungen zum Begriff der distributiven Gerechtigkeit. Abschließen werde ich mit einigen Fragen, die sich einer philosophischen Theorie globaler Gerechtigkeit heute stellen.

\section{Probleme des kosmopolitischen Liberalismus}

Angesichts des Kolonialismus und seiner Folgen, des Vietnam-Kriegs, des internationalen Wohlstandsgefälles, der wirtschaftspolitischen Forderungen der südlichen Länder, vor allem aber von Hunger und Armut in diesen Ländern sieht sich die politische Philosophie spätestens seit den 70er Jahren vor die Aufgabe gestellt, Prinzipien globaler Gerechtigkeit zu bestimmen. Einige Autoren haben sich von John Rawls' Gerechtigkeitstheorie inspirieren lassen, seine Ausgangshypothese einer Gesellschaft als geschlossenes System (Rawls, 1979, S. 24) indes kritisiert und eine globale Anwendung des Vertragsmodells angeregt (vgl. Schaber, 1991, S. 67-85). Charles Beitz hält angesichts globaler ökonomischer Abhängigkeiten die Hypothese nationalstaatlicher Autarkie für unhaltbar. Sind natürliche Ressourcen, Einkommen und Wohlstand zwischen Ländern ungleich verteilt, so müssen ihm zufolge Prinzipien der internationalen distributiven Gerechtigkeit bestimmt werden, die primär auf Menschen, nicht auf Staaten anzuwenden sind. Beitz bezeichnet seine Konzeption als kosmopolitisch; sie befasst sich mit moralischen Beziehungen zwischen Mit- 
gliedern einer universellen Gemeinschaft, für welche Staatsgrenzen von zweitrangiger Bedeutung sind. Falls ökonomische, politische und kulturelle Beziehungen ein globales Kooperationssystem erzeugen, können Bürger eines Staates Gerechtigkeitsverpflichtungen gegenüber Bürgern anderer Staaten haben, so sein Argument.

$\mathrm{Ob}$ Kooperation gerechtigkeitsrelevant ist und, falls ja, unter welchen Bedingungen, ist umstritten. Rawls (1979, S. $148 \mathrm{ff}$ ) versteht Gesellschaften als Unternehmen der Zusammenarbeit zum gegenseitigen Vorteil. Die normativen Implikationen dieser Annahme sind relativ bescheiden. Moralische Verpflichtungen zur fairen Verteilung von Gewinn und Verlust lassen sich nicht bereits dann begründen, wenn einige Menschen von der Arbeit anderer Menschen profitieren, sondern erst dann, wenn die Gewinner ein rationales Interesse daran haben, die Verlierer zu entschädigen, weil sie damit rechnen, irgendwann auf deren Unterstützung angewiesen zu sein, und wenn die Verlierer damit rechnen, noch schlechter dazustehen, wenn sie nicht kooperieren. Internationaler Handel stellt, wie Brian Barry gegen Beitz geltend macht, kein echtes Kooperationsystem dar, das Fairnessregeln hervorbringt; Umverteilung ist für Reiche nicht im gleichen Maß vorteilhaft wie für Arme. Kosmopolitischen Theorien liegen denn auch häufig nicht Kooperationsmodelle zugrunde, sondern Kants Idee moralischer Reziprozität (Richards, 1982), die es Menschen verbietet, andere Menschen als Mittel zum Zweck ihres eigenen Vorteils zu betrachten. Eine realistischere Variante des Kosmopolitismus vertritt Barry (1989), wenn er zu bedenken gibt, die gerechte Gestaltung der Weltordnung setze die Herausbildung durchsetzungsfähiger internationaler Institutionen voraus. Da sich die Ungerechtigkeit der Weltwirtschaftsordnung ihm zufolge in aggressiven Praktiken multinationaler Unternehmen und ihrer Heimatstaaten gegenüber Entwicklungsländern manifestiert, verteidigt er den Anspruch armer Länder auf staatliche Souveränität über die natürlichen Ressourcen. Gegenstand der Gerechtigkeit ist gemäß seiner Sichtweise die Verteilung der Kontrolle über materielle und nicht-materielle Ressourcen, letztlich die Verteilung von Handlungsfreiheit oder Macht ${ }^{1}$.

Die Frage nach der Gerechtigkeitsrelevanz des Verhaltens mächtiger staatlicher und nicht-staatlicher Akteure wird in manchen Theorien globaler Gerechtigkeit verdrängt. Die Meinung, für ihr Wohlstandsniveau seien Staaten selbst verantwortlich, die prekäre Situation armer Länder sei primär auf internes Politikversagen zurückzuführen (Höffe, 1999, S. 409 ff), ist verbreitet. Ein weiterer Einwand der Kosmopolitismus-Kritik lautet, Forderungen nach Verteilungsgerechtigkeit könnten sinnvollerweise nur im Rahmen funktionierender rechtlich-politischer Institutionen erhoben werden. Tatsächlich setzt die Erfüllung globaler Gerechtigkeitsforderungen weltstaatlich funktionierende Institutionen voraus, und es ist erstaunlich, dass einige kosmopolitische Philosophen sich dieser Konsequenz verschließen

1 „If we understand ,resources' in a very wide sense, so that it includes all kinds of rights to act without interference from others, to constrain the actions of others, and to bring about changes in the non-human environment, then we can say that the subject-matter of justice [...] is the distribution of control over material resources. At this high level of generality, it is complemented by the principle of equal liberty, which is concerned with the control over non-material resources. To put it in a slogan [...]: humanity is a question of doing good whereas justice is a question of power." Barry, 1989, S. $456 \mathrm{f}$. 
(Chwaszcza, 1996, S. 173 ff), wobei immerhin zu erwähnen ist, dass sie institutionelle Fragen nicht einfach ausblenden (Pogge, 1992; Beitz, 1994). Wolfgang Kersting anerkennt zwar den impliziten Kosmopolitismus des Kontraktualismus, lehnt die Hypothese eines globalen Urzustands aber ab, weil sie notwendig zur Begründung eines Weltstaats führt, der ihm zufolge die Gestalt eines despotischen Weltsozialstaats annehmen müsste (Kersting, 1997, S. 262-297). Als Rezept zur Bekämpfung der internationalen Ungerechtigkeit empfiehlt Kersting dagegen eine „Politik der strukturellen Zivilisierung “, die auf die Förderung von Marktwirtschaft, Rechtstaatlichkeit und Demokratie in armen Ländern zielt (Kersting, 1997, S. 343). Rawls möchte aus der liberalen Völkerrechtsgemeinschaft neben gesetzlosen Staaten auch Gesellschaften ausschließen, die aus historischen oder ökonomischen Gründen nicht zu wohlgeordneten Völkern werden können. Er bejaht immerhin eine Beistandspflicht diesen Gesellschaften gegenüber, die sich jedoch nur auf den Aufbau gerechter Institutionen und die Förderung autonomer Handlungsfähigkeit der Staaten, nicht auf Armutsbekämpfung beziehen soll. Die globale Anwendung distributiver Gerechtigkeitsprinzipien verbietet sich in dieser Perspektive (Rawls, 1999, S. 105-120; vgl. Beitz, 2000).

Als Alternative zur gleichheitsorientierten Verteilungsgerechtigkeit postuliert Kersting neuerdings (Kersting, 2002, S. 97-142) eine suffizienzorientierte Verteilungsgerechtigkeit, die auf Subsistenzsicherung zielt, aber nicht als Korrektur einer Kooperationsungerechtigkeit verstanden werden darf. In den Genuss suffizienzorientierter Verteilungsgerechtigkeit können nur Länder kommen, die international nicht mehr kooperieren. Ihre Notlage darf nicht auf die ungerechte Verteilung globaler Kooperationsgewinne und -lasten zurückgeführt werden, sondern muss ihre Ursache in „Naturkatastrophen, politisch induzierten Hungersnöten, Bürgerkriegen und domestischen politischen und ökonomischen Strukturmängeln“ haben (Kersting, 2002, S. 111). Problematisch an diesem Ansatz ist die Weigerung, mögliche Ungerechtigkeiten der wirtschaftlichen Kooperation auch nur in Betracht zu ziehen. Überdies wird es in einer Welt von „failed states“ und ruinösen Wirtschaftskrisen schwer fallen, sich auf Kriterien zu einigen, die erfüllt sein müssen, damit die Staatengemeinschaft ihre Beistandspflicht anerkennt. Bemerkenswert ist der Ansatz hingegen, weil er, ausgehend von einer Kritik der kantianischen Orthodoxie, ein Subsistenzrecht sowie ein Individualrecht auf Entwicklung der Fähigkeiten durch Bereitstellung von Ausbildungssystemen begründet.

Die Ansicht, fehlende Entwicklungschancen von Staaten seien selbstverschuldet, ist so unhaltbar wie jede Ideologie, die Reichtum und Armut ausschließlich auf persönliches Verdienst oder Verschulden zurückführt. Korruptes Verhalten setzt trivialerweise korrumpierendes Verhalten voraus; es ist eine Folge der innerund zwischenstaatlich ungleichen Verteilung von Macht, Ressourcen und Handlungsmöglichkeiten, die Abhängigkeiten schafft und vergrößert. Die Kritik an demokratischen und menschenrechtlichen Defiziten armer Länder könnte an Glaubwürdigkeit gewinnen, wenn sie die Frage, inwieweit die kritisierten Staaten im System globaler Abhängigkeit überhaupt selbstbestimmt agieren können, nicht verdrängen würde. Als besonders fragwürdig erweist sich die Rede von hausgemachten Notlagen angesichts der vielfältigen Auswirkungen einer Weltwirtschaft, 
worin bereits kurzfristige Entscheide von Anlegern ganze Volkswirtschaften ruinieren können.

Kosmopolitische Theorien weisen also zu Recht auf die komplexen Ursachen des globalen Wohlstands- und Demokratiegefälles hin. Kritisierbar sind sie in dem Maße, wie sie die politisch-institutionellen Bedingungen der Erfüllung ihrer Postulate nicht thematisieren. Gleiche Ausgangsbedingungen für alle Menschen vermag nur eine demokratisch-föderalistische Weltregierung $\mathrm{zu}$ garantieren, so argumentieren radikale Vertreter des Kosmopolitismus (Nielsen, 1988). Für Marxisten wie Kai Nielsen versteht sich dabei aber von selbst, dass unter kapitalistischen Verhältnissen eine solche Vision utopisch bleiben muss. Beitz hingegen glaubt, ein moralischer Kosmopolitismus sei weder gleichbedeutend mit einem politisch-institutionellen Kosmopolitismus noch impliziere er diesen (Beitz, 1994, S. 124 f). Der moralische Kosmopolitismus zielt meist auf ein System der internationalen Umverteilung mittels Besteuerung; reiche Leute, wo auch immer sie leben, sollen zugunsten armer Leute, wo auch immer sie leben, besteuert werden. Die Einrichtung eines solchen Steuersystems erfordert massive Eingriffe in die staatliche Souveränität. Überdies ist selbst dann, wenn sich Umverteilungsmechanismen zwischen Staaten eingespielt haben, damit zu rechnen, dass nur reiche Oberschichten in armen Ländern davon profitieren und vor allem ärmere Unterschichten in reichen Ländern dafür bezahlen (Barry, 1998, S. 153). Offen bleibt, wie die Probleme, auf die der moralische Kosmopolitismus zu Recht hinweist, anders zu lösen sind als durch globale Institutionen, die über weitgehende Kompetenzen, ausreichende Informationen, Sanktionsmöglichkeiten und demokratische Legitimation verfügen.

Thomas Pogge, ein weiterer Vertreter des Kosmopolitismus, versteht Ungleichheit als Resultat eines globalen Systems ökonomischer, rechtlicher und politischer Institutionen, worin alle Menschen koexistieren und das Verhalten der Bessergestellten die Lebensumstände der Schlechtergestellten beeinflusst. Solche Institutionen, die die Verteilung sozialer Güter regeln und moralisch bedeutsam sind, sind nicht naturgegeben und unveränderbar, sondern werden von ihren Nutznießern verantwortet. Konkret manifestiert sich die Ungerechtigkeit im Ausschluss eines großen Teils der Weltbevölkerung von der Verfügung über natürliche Ressourcen. Pogges Reformvorschlag zielt auf eine globale Rohstoffdividende (Pogge, 1998a), die den ärmsten Menschen einen fairen Anteil an der Ressourcennutzung sichern soll, nicht den ärmsten Staaten, wobei die Umverteilung offiziellen und inoffiziellen internationalen Organisationen zu übertragen wäre. Unter Verweis auf Artikel 28 der Allgemeinen Erklärung der Menschenrechte, wonach jeder Mensch Anspruch auf eine soziale und internationale Ordnung hat, in welcher die in der Erklärung angeführten Rechte und Freiheiten vollumfänglich verwirklicht werden können, definiert er Menschenrechte als Ansprüche an soziale Institutionen und deren Nutznießer (Pogge, 1998b). Er zählt dazu zwar negative wie positive Freiheitsrechte, betont aber, dass auch letztere bloß eine negative Verpflichtung implizieren. Die negative Rechtspflicht besteht darin, nicht an der Durchsetzung ungerechter Institutionen mitzuwirken. Faktisch kann sich die Erfüllung dieser Pflicht freilich nicht in Enthaltung 
oder Unterlassung erschöpfen. Die Nutznießer der bestehenden Verteilungsordnung verletzen die negative Rechtspflicht nämlich bereits dann, wenn sie „nicht nach Kräften auf die erforderlichen institutionellen Veränderungen hinarbeiten“ (Pogge, 1998b, S. 381). Die moralische Pflicht der Wohlhabenden besteht in Pogges Sicht nicht im Beistand, wie Rawls vorschlägt, sondern im Verzicht darauf, eine Weltordnung anderen aufzuzwingen (Pogge, 2001).

Es bleibt der Eindruck, der Abstand zwischen Anhängern und Kritikern² des Kosmopolitismus sei geringer als vermutet. Während die Anhänger sich der Einsicht nicht verschließen können, dass globale Ungerechtigkeit eher durch Machtdisparitäten denn durch ungleiche Güterverteilung verursacht wird (vgl. Forst, 2001, S. 176 f), beruft sich ein Gegner wie Kersting auf Marx’ Kritik der strukturerhaltenden Verteilungspolitik der Sozialdemokratie und macht geltend, der sozialdemokratische Kosmopolitismus unterstelle grundlos die Möglichkeit eines globalen kapitalistischen Konsenses und ziehe systembewahrende Verteilungsreform struktureller Veränderung vor (Kersting, 1997, S. 301 f; 2002, S. 101 f). Mit guten Gründen weist der Kosmopolitismus nationalistische und kommunitaristische Mythen zurück (Barry, 1999; Satz, 1999). Verteilungspolitik setzt keine Wertegemeinschaft voraus, sondern die Fähigkeit jener, die einen größeren Anteil an einem bestimmten Gut beanspruchen, sich politisch zu organisieren und zu artikulieren. Dass globale Gerechtigkeit die Reform bestehender oder die Errichtung neuer Institutionen auf Weltebene voraussetzt, wird in neueren kosmopolitischen Ansätzen durchaus eingeräumt (vgl. Jones, 1999, S. 227 ff; Moellendorf, 2002, S. 171 ff; Hurrell, 2001). Kerstings Idee eines basale Rechte auf Existenz, Subsistenz und Entwicklung umfassenden Menschenrechtsuniversalismus könnten kosmopolitische Theoretiker beipflichten (vgl. Beitz, 1999; Jones, 1999, S. 50-84). Beide Positionen bleiben indes unbefriedigend. Solange Kritiker des Kosmopolitismus normativ relevante Folgen der sich globalisierenden Wirtschaft nicht zur Kenntnis nehmen, sind ihre Vorschläge kaum hilfreich. Anhänger des Kosmopolitismus sprechen zwar zu Recht von negativer Verantwortung, müssen aber erst konkretisieren, was darunter zu verstehen ist: Was sollen Regierungen unterlassen, was Unternehmer und Anleger, was Konsumenten, um diese Verantwortung wahrzunehmen? Wie sind internationale Institutionen zu verändern, damit darin auch den Stimmen schwächerer Akteure Gewicht zukommt? Und unter welchen Bedingungen ist die private Verfügungsmacht über ökonomische Mittel mit liberalen Gerechtigkeitsvorstellungen kompatibel?

\section{Der Streit um wirtschaftliche Souveränität}

Die Politik, die seit drei Jahrzehnten die Weltwirtschaftsordnung umgestaltet, wird mit der Behauptung gerechtfertigt, grenzenlose Handels- und Investitionsfreiheit vermöge weltweit Wohlstand für alle und genügend Arbeitsplätze zu

2 Gemeint sind wohlverstanden nur liberale, nicht nationalistische, kommunitaristische und kulturrelativistische Kritiker des Kosmopolitismus. 
schaffen. Zwar hat die Freihandelslehre die internationale Wirtschaftspolitik in der gesamten Nachkriegszeit maßgeblich geprägt. Doch die Einsicht, dass im globalen Wettbewerb die Ausgangsbedingungen nicht für alle Akteure gleich sind und dass es für ökonomisch schwächere Länder schwierig ist, die Industrialisierung unter Verzicht auf protektionistische Maßnahmen nachzuholen, hat im Rahmen der UNO Vorstöße inspiriert, die auf die Verbesserung der Entwicklungschancen ärmerer Länder zielten. Als Entwicklungsparadigma (Altvater/Mahnkopf, 1999, S. 134-145) hat sich dann aber der Neoliberalismus durchgesetzt, nicht weil er weltweit konsensfähig ist oder wissenschaftlich überzeugt, sondern weil er mächtigen Interessen zum Ausdruck verhilft.

Projekte zur Errichtung einer neuen internationalen politischen, ökonomischen und sozialen Ordnung, wie sie in der Nachkriegszeit ausgearbeitet und partiell auch realisiert worden sind, beruhten auf zwei Grundsätzen (vgl. Kapstein, 1999). Da sich sowohl die protektionistische Abkapselung wie auch die weitgehende Deregulierung der Wirtschaft als verhängnisvoll erwiesen hatten, wurden Freihandel wie Wohlfahrtsstaaten für nötig erachtet. Während für die wirtschaftliche Entwicklung internationale Organisationen wie das Allgemeine Zoll- und Handelsabkommen (GATT), die Weltbank und der Internationale Währungsfonds (IMF) zuständig waren, blieb die Förderung sozialer Gerechtigkeit den Staaten überlassen, die zu diesem Zweck über eine gewisse währungsund sozialpolitische Autonomie verfügen mussten. Angesichts der Erfahrung, dass weltweiter Freihandel innerhalb wie zwischen Staaten Gewinner und Verlierer schafft, stieß das Vorhaben, national wie international korrigierend und ausgleichend einzugreifen, zunächst auf breite Zustimmung. Die Gründung der Welthandels- und Entwicklungskonferenz (UNCTAD, 1962) entsprach den Anliegen der Entwicklungsländer, die sich 1974 im Programm einer neuen Weltwirtschaftsordnung (NIEO) deutlicher artikulierten (vgl. Marchand, 1994; Bello, 1998). Das Programm zielte auf einen internationalen Ausgleich mittels besserem Marktzugang, Technologietransfer, Rohstoffpreisabkommen, verstärkter Entwicklungshilfe und Entschuldung; es war aber auch Ausdruck eines bestimmten Völkerrechtsverständnisses.

Obgleich gemäß Völkerrechtslehre nur Staaten Schöpfer von Völkerrecht sind, zeichnet sich seit dem 19. Jahrhundert eine neue Entwicklung ab. Multinationale Unternehmen können die handelsrechtliche Ausgestaltung nationaler Gesetzgebungen entscheidend mitbestimmen, sie können sogar durch Verträge über langfristige Investitionsabkommen mit Staaten neue Völkerrechts-Regeln erzeugen und bei Vertragsverletzung durch das Gastland dank Intervention des Herkunftslandes durchsetzen (Muchlinski, 1997). Versuche ausländischer Unternehmen, auf diesem Weg die Gesetzgebung des Gastlands zu beeinflussen, haben in vielen Fällen zu massivem Machtmissbrauch geführt. Bemühungen seitens der UNO, politische Aktivitäten von multinationalen Unternehmen in den Gastländern zu beschränken, sind gescheitert. Ein UN-Entwurf zu einem Verhaltenskodex für transnationale Unternehmen sah vor, dass diese sich nicht in die inneren Angelegenheiten des Gastlandes einmischen dürfen, die einheimischen Gesetze auch dort respektieren, wo sie regulierend eingreifen, die wirtschaftlichen und entwicklungs- 
politischen Zielsetzungen teilen und in ihrem Geschäfts- und Produktionsverhalten die soziokulturellen Verhältnisse nicht stören (Muchlinski, 1997, S. 90 ff). Gegen solche Bestimmungen haben die multinationalen Unternehmen mit Erfolg lobbyiert. Obgleich auch die Heimatstaaten vergleichbarem Druck auf die Gesetzgebung ausgesetzt sind, setzen sie sich in der Regel dafür ein, globale Wirtschaftspolitik und globales Wirtschaftsrecht nach Maßgabe der Interessen ihrer Unternehmen $\mathrm{zu}$ gestalten. Ein Beispiel staatlicher Intervention im Interesse der Unternehmen sind bilaterale Abkommen zum Schutz und zur Förderung von Investitionen. Versuche der Unternehmen, ein multilaterales Investitionsschutz-Abkommen durchzusetzen, sind bislang allerdings gescheitert. Initiativen dieser Art wurden seit den 30 er Jahren ergriffen, seit den 60er Jahren wird das Anliegen von der OECD, neuerdings von der WTO vertreten.

Die von den Industrieländern sabotierten Bemühungen um die Kontrolle transnationaler Unternehmen gehen zurück auf das UNO-Programm zur Errichtung einer Neuen Weltwirtschaftsordnung. Dessen Ziel war die Stärkung der wirtschaftlichen Souveränität der südlichen Staaten (G77). Im Gegenzug veröffentlichte die OECD 1976 die Richtlinien für multinationale Unternehmen, die größere Investitionsfreiheit und Schutz der Vertrags- und Eigentumsrechte forderten. Die in der Gruppe der 77 organisierten Länder vermochten ihre Vorstellungen in der Folgezeit nicht durchzusetzen. Die UNO musste die wirtschaftspolitische Definitions- und Entscheidungsmacht an GATT und WTO abtreten. Mittlerweile wird nicht mehr eine neue internationale Wirtschaftsordnung, sondern eine neue Weltordnung für Handel und Investition angestrebt (Muchlinski, 1997, S. $95 \mathrm{ff})$.

Wie sind diese beiden Ordnungen unter normativen Gesichtspunkten zu beurteilen? Die NIEO war Ausdruck einer Gerechtigkeitskonzeption, die internationale Umverteilung von Wohlstand und mehr Entscheidungsmacht für ärmere Länder in internationalen Organisationen verlangte. Die Überwindung der kapitalistischen Weltordnung war nicht beabsichtigt. Das Programm der NIEO wurde teils von Regimes unterstützt, die demokratische Partizipation und Kontrolle über die Sozialpolitik verhinderten. Politisches Gewicht konnte ihm vorübergehend nur zukommen, weil einige ärmere Länder über Ressourcen verfügten, die die Industriestaaten begehrten. Die Öl-exportierenden Länder besaßen dank ihrer Fähigkeit, die Preise zu erhöhen, Verhandlungsmacht, die sie jedoch in den 80er Jahren ebenso verloren wie ihre Fähigkeit, kollektiv zu agieren. Der Siegeszug neoliberaler Ideologien führte dann zur Delegitimierung distributiver Politik (Cason, 2000). Unter normativen Aspekten ist zunächst nur der Umstand relevant, dass es offenbar möglich ist, auch Fragen globaler Gerechtigkeit, die sich auf die Wirtschafts- und Sozialpolitik beziehen, in der Sprache des Völkerrechts zu erörtern. Die Vision einer neuen Weltwirtschaftsordnung gründet auf Prinzipien der neuen Völkerrechtsordnung (Kimminich, 1997, S. 305-318). Der Anspruch auf souveräne Gleichheit aller Staaten und das Selbstbestimmungsrecht der Völker wird auch im Bereich der Wirtschaft erhoben; daraus lassen sich das Prinzip der Souveränität über die natürlichen Ressourcen und das Recht der Staaten auf Kontrolle ausländischer Investoren herleiten. 
GATT und WTO haben eine konfliktreiche Geschichte. Die Organisation anerkennt zwar formell das Prinzip der souveränen Gleichheit der Staaten, ihre konsensorientierten Entscheidungsverfahren stellen aber im Urteil von Kritikern nichts anderes als organisierte Heuchelei dar (Steinberg, 2002): Angesichts des Beitritts zahlreicher Entwicklungsländer seit den späten 50er Jahren haben die Industriestaaten Strategien entwickelt, die es ihnen erlauben, unter Ausnützung ihrer größeren Verhandlungs- und Sanktionsmacht ihre Hegemonie zu behaupten, ohne Verfahrensregeln zu verletzen. Sie bestimmen bis heute die Tagesordnung der Verhandlungsrunden und können ihre Anliegen in der Regel gegen jene der ärmeren Länder durchsetzen, wie etwa der anhaltende, bislang aber folgenlose Widerstand dieser Länder gegen das Abkommen über handelsrelevante Aspekte geistiger Eigentumsrechte (TRIPs) zeigt. Die Ergebnisse der Runden sind, so die Konklusion von Richard Steinberg, Abbild des Machtungleichgewichts zwischen Industrieund Entwicklungsländern und werden letzteren, obwohl sie formell gleichberechtigt sind, faktisch aufgezwungen.

Die Politik von WTO, IWF und Weltbank erweckt generell den Eindruck paternalistischen Verhaltens reicher gegenüber armen Staaten. Ein moderater Kritiker wie Joseph Stiglitz spricht von globaler Politikgestaltung ohne globale Regierung. Sie wird im Rahmen dieser Institutionen von wenigen staatlichen und privaten Akteuren beschlossen, die den von ihren Entscheidungen Betroffenen keine Rechenschaft schulden; diese verfügen ihrerseits in ihrer Mehrheit über kein Mitspracherecht. Hinzu kommt nach Ansicht des ehemaligen Chefökonomen der Weltbank der Umstand, dass den Entscheidungen weder ökonomische noch politische Rationalität zugrunde liegt, sondern eine Mischung aus Ideologie, Sonderinteressen und schlechter Ökonomie (Stiglitz, 2002, S. 12, 36). Als Fazit ergibt sich somit, dass in einer von den mächtigen Industriestaaten mit Hilfe internationaler Handels- und Finanzorganisationen diktierten globalen Wirtschaftspolitik Selbstbestimmung und Wahlfreiheit für die Mehrheit der Staaten zur Fiktion wird. Werden dem Prinzip vollständiger Handels- und Investitionsfreiheit alle anderen normativen Kriterien untergeordnet, dann wird der Anspruch auf staatliche Souveränität offenkundig verletzt.

Werden Legitimitätskriterien der globalen Wirtschaftsordnung in der Sprache des Völkerrechts definiert, so ist als ungerecht eine Wirtschaftspolitik zu beurteilen, die die Souveränität von Staaten verletzt. Dieser Auffassung liegt ein traditionelles Völkerrechstsverständnis zugrunde, das zunehmend hinterfragt wird. Die Relativierung des Souveränitätsprinzips ist in neueren Völkerrechtskonzeptionen nicht zwingend ungerecht. Im Hinblick auf die Gestaltung der Weltwirtschaftsordnung wären folgende Punkte zu berücksichtigen:

- Die Gleichheit und autonome Handlungsfähigkeit aller Staaten ist auch im Bereich der Wirtschafts- und Sozialpolitik zu respektieren. Dies gilt unter der Bedingung, dass die Staaten ihrerseits die Freiheitsrechte ihrer Bürgerinnen und Bürger einschließlich wirtschaftlicher und sozialer Rechte garantieren. Ob ein Recht auf Handel und Investition bedingungslos zugestanden werden kann, hängt davon $\mathrm{ab}$, ob es universalisierbar ist. Zu beurteilen ist, ob dieses in der Regel eher von mächtigen Akteuren genutzte Recht mit den gleichen Rechten aller Akteure, Han- 
del zu treiben, zu investieren, zu produzieren, zu subsistieren, Zugang zu lebensnotwendigen Ressourcen und öffentlichen Gütern zu haben, vereinbar ist. Erfahrungsgemäß sind im Bereich wirtschaftlicher und sozialer Freiheiten Konflikte zwischen konkurrierenden Rechtsansprüchen unausweichlich, eine Rangordnung ist daher nötig.

- Menschen dürfen gemäß liberalem Verständnis unter der Bedingung, dass sie die gleiche Freiheit der andern achten, in der Wahl und Ausführung ihrer Lebenspläne nicht bevormundet werden, das gilt auch für die Verfolgung materieller Interessen. Ihre individuelle Wahlfreiheit stößt dort auf Grenzen, wo die Ausführung des Lebensplans unzumutbare Auswirkungen auf die Freiheit anderer Menschen hat; bei der Verfolgung ihrer materiellen Interessen dürfen sie die wirtschaftlichen Handlungsmöglichkeiten anderer Menschen nicht zerstören. Globale Wirtschaftspolitik wäre dann gerecht, wenn sie die Freiheit aller Menschen, selbst gewählte Lebenspläne zu verwirklichen, garantieren könnte. Jeder paternalistischen Einflussnahme, die einen bestimmen Lebensplan beziehungsweise eine bestimmte wirtschaftliche Wertordnung aufzwingt, hätte sie sich zu enthalten. Überdies hätte sie darüber zu wachen, dass die Regeln fairer Kooperation nicht verletzt werden, dass alle Menschen vom wirtschaftlichen Kooperationssystem profitieren und dessen Lasten gemeinsam tragen. Im Streit darüber, ob die WTO als Keimform einer Weltverfassung gelten kann und ob in dem Falle auch Sozialrechte und Umweltklauseln festzuschreiben sind, geht es letztlich um solche Regeln.

- Von einem kosmopolitischen Standpunkt her betrachtet ist es plausibel, zusätzlich zur gleichen Autonomie demokratisch legitimierter Staaten die Verbesserung der Startpositionen schlechter gestellter Staaten zu postulieren, da schlechte Ausgangspositionen eine autonome Politik erschweren. Die Chancen solcher Staaten könnten freilich durch den Schutz der negativen Freiheitsrechte aller Menschen ungeachtet ihrer nationalen Zugehörigkeit bereits verbessert werden. Nicht nur Heimatstaaten, auch andere Staaten oder transnationale Unternehmen können Menschen dazu zwingen, etwas unfreiwillig zu tun. In einigen Fällen ist die Verletzung negativer Freiheitsrechte offenkundig, etwa bei der entschädigungslosen Vertreibung von Kleinbauern oder bei Verstößen gegen die Menschenwürde, bei Zwangsarbeit und Gewalt gegen Frauen in Export-Sonderzonen. Eindeutig ist die Situation auch dann, wenn Unternehmen von Rechtsverletzungen durch Militärdiktaturen profitieren. Weitere Fälle sind schwieriger zu beurteilen. Kommt die Patentierung biologischer Ressourcen einer Enteignung der ursprünglichen Produkteure gleich? Kommen Strukturanpassungsprogramme, die finanzielle Hilfe an die Auflage binden, der Staat müsse seine Märkte öffnen und sich aus seiner sozial-, bildungs- und gesundheitspolitischen Verantwortung zurückziehen, der Ausnutzung einer Notlage gleich? Wie auch immer die Antwort ausfallen mag, gewiss ist, dass die Einschränkung der wirtschafts- und sozialpolitischen Handlungsfähigkeit demokratischer Staaten und ihrer Bürgerinnen und Bürger liberalen Gerechtigkeitsintuitionen zuwiderläuft. Dies gilt erst recht für die neuerdings vermehrt angewandte Praxis, wirtschaftliche Hilfe oder Handelserleichterungen an die Bereitschaft des interessierten Landes zu knüpfen, seine Politik den Wünschen einer Großmacht unterzuordnen. 


\section{Die Verteilung der Freiheit}

Das Gerechte ist eine Zuteilung von Sachen an Menschen, bei der Gleiche Gleiches erhalten. Diese Auffassung scheint unbestritten zu sein, wie Aristoteles ${ }^{3}$ konstatiert. Die politische Philosophie geht von dieser Begriffsbestimmung aus und wendet sich dann ihrer eigentlichen Aufgabe zu. Sie muss untersuchen, worin die Gleichheit und Ungleichheit der Menschen besteht. In der Aufgabenstellung kommt das komplexe Verhältnis zwischen Gleichheit und distributiver Gerechtigkeit in Aristoteles' Theorie deutlich zum Ausdruck. Gerechtigkeit besteht in der Achtung des Gesetzes und der bürgerlichen Gleichheit, das heißt der gleichmäßigen Verteilung der Güter. Gerechtes Handeln zielt auf das Glück des Gemeinwesens. Distributive Gerechtigkeit regelt die Verteilung von Gütern oder Werten, die den Bürgern eines politischen Gemeinwesens zustehen. Zwar setzt Aristoteles Gerechtigkeit mit Gleichheit gleich, die gerechte, mithin gleiche Verteilung muss jedoch einem bestimmten Wert angemessen sein, das Maß des zu Verteilenden muss dem Wert des Empfängers entsprechen. Welcher Wert Bemessungsgrundlage sein und wer in den Genuss des gleichmäßig zu verteilenden Gutes kommen soll, ist abhängig von der jeweiligen politischen Ordnung.

Das Prinzip „Jedem das Seine“ besagt, dass das Recht tatsächlich als Gleichheit $\mathrm{zu}$ begreifen ist, freilich nicht als Gleichheit für alle, sondern als Gleichheit für Gleiche. Worin die Menschen gleich sein müssen, um einen gleichen Anteil eines bestimmten Gutes beanspruchen zu können, ob dabei Herkunft, Reichtum, freie Geburt oder Tugend ausschlaggebend sein sollen, bleibt stets zu prüfen und hängt auch davon ab, welche Aufgabe dem politischen Gemeinwesen zugeschrieben wird. Wird dieses nicht als militärisches oder ökonomisches Zweckbündnis verstanden, sondern als Vereinigung mit dem Ziel, gut und glücklich zu leben, muss Bildung und Tugend der Vorrang zukommen. Die beste Staatsverfassung ist jene, worin alle glücklich leben. Distributive Gerechtigkeit besteht somit darin, alle in den Stand zu versetzen, ihre Anlagen so zu entwickeln, dass sie glücklich leben können. Als eine Gemeinschaft Gleichberechtigter ist der Staat zugleich eine pädagogische Institution; die von der Politik zu Erziehenden aber sind bezüglich ihrer Anlage zur Tugend nicht gleich. Politisch-pädagogische Kompetenz kommt jenen zu, die zur Entwicklung wahrer Tugend fähig sind. Die Aufgabe distributiver Politik ist es, äußere Güter so zu verteilen, dass auch den weniger edlen Naturen der Weg zum guten Leben geebnet wird, vor allem aber, die Menschen zur Tugend zu erziehen. Distributive Gerechtigkeit im aristotelischen Sinne erweist sich somit als ein kompliziertes Unternehmen, das mit pädagogischen Mitteln versucht, die natürliche Ungleichheit jener Menschen, die als Staatsbürger in Betracht kommen, zu korrigieren, um die gleichberechtigten Staatsbürger zu befähigen, das Ziel der Politik auch individuell zu verwirklichen. Als pädagogisches Projekt setzt distributive Politik notwendig die Ungleichheit zwischen Erziehern oder Gesetzgebern und zu Erziehenden voraus; jenen, die über hinreichende ethisch-politische Kom-

3 Vgl. zum Folgenden Aristoteles 1981, Politik 1280a 10-1283a 29, 1324a 22 ff, 1328a 25-1329a 1, 1331b 40-1332b 10; Aristoteles 1985, Nikomachische Ethik 1129a-1131b. 
petenz verfügen, obliegt es, darüber zu entscheiden, welche Verteilung das gute Leben am ehesten fördert.

Auch der modernen Idee von Gerechtigkeit und Recht liegt der Gedanke der Verteilung zugrunde. Diese Behauptung scheint dem modernen Grundsatz zu widersprechen, wonach die Aufgabe der Politik in der Herstellung von Sicherheit zwischen formal gleichen Individuen besteht. Verteilung zielt dann nicht mehr auf die Erziehung zum guten Leben, sondern auf Sicherheit mittels der Definition klarer Eigentumsrechte. Distributive Gerechtigkeit darf sich, so Klaus Günther, nur auf Rechtssubjekte beziehen, die wechselseitig ihr Recht auf gleiche Freiheit anerkennen, und sie darf nur im Zuge einer Gesetzgebung bestimmt werden, an der alle Betroffenen gleichberechtigt teilnehmen. Gleiche negative und aktive Freiheitsrechte können nicht selbst Gegenstand der Verteilung sein, sondern definieren den Rahmen jedes möglichen Systems distributiver Gerechtigkeit. Als Legitimitätskriterium politischer Ordnung tritt damit distributive Gerechtigkeit zurück (Günther, 1994, S. 160, 167 f). Dies trifft freilich nur dann zu, wenn unter distributiver Gerechtigkeit die Verteilung von Gütern oder Chancen für die Verwirklichung des guten Lebens verstanden wird. Kant jedoch setzt den Begriff mit Gesetzgebung gleich.

Den Naturzustand definiert Kant ${ }^{4}$ als Zustand, „in welchem keine austeilende Gerechtigkeit ist“, und setzt ihm den Zustand „einer unter einer distributiven Gerechtigkeit stehenden Gesellschaft“ entgegen. Das klassische Prinzip, wonach jedem das Seine zu geben sei, ist für Kant ungereimt; es muss übersetzt werden in die Aufforderung, in einen Zustand zu treten, „worin jedermann das Seine gegen jeden anderen gesichert sein kann“. Der rechtliche Zustand entsteht im Zuge der reziproken Zusicherung des Gewaltverzichts. Das natürliche Recht aller auf alle verfügbaren Güter gerät notwendig in Konflikt mit dem Anspruch aller auf Freiheit; die unbegrenzte Freiheit widerspricht dem gleichen Recht auf Freiheit. Recht wird somit definiert als „der Inbegriff der Bedingungen, unter denen die Willkür des einen mit der Willkür des andern nach einem allgemeinen Gesetze der Freiheit zusammen vereinigt werden kann“. Der Begriff hat nichts zu tun mit Bedürfnissen und Interessen, er bestimmt nicht die Zwecke menschlichen Lebens und die Mittel, sie $\mathrm{zu}$ erreichen. Recht bezeichnet ausschließlich die Begrenzung individueller Freiheit zwecks Garantie gleicher individueller Freiheit. Nur dieses Recht, der Gewalt der anderen nicht ausgesetzt zu sein, ist angeboren, steht also allen Menschen als Menschen zu.

Das Recht darf, so lautet auch die klassisch-liberale Position, nicht im Dienst der Verwirklichung des guten Lebens stehen, sondern hat ausschließlich dafür zu sorgen, dass alle Menschen in Sicherheit leben und ihre Konzeption des guten Lebens frei wählen können. Es gilt der Vorrang des Rechten vor dem Guten, das heißt der Vorrang negativer Freiheiten sowohl vor ethischen Idealen als auch materiellen Ansprüchen. Die Vorrangregel anerkennt auch der moderne, egalitäre Liberalismus, interpretiert sie aber differenzierter. Er bestreitet nicht, dass dem Prinzip der gleichen Freiheit unbedingter Vorrang vor jeder Art ideellen und

4 Vgl. zum Folgenden Kant, AA Bd. 6, S. 230, 237, 306, Metaphysische Anfangsgründe der Rechtslehre; AA Bd. 8, S. 289 f, Über den Gemeinspruch. 
materiellen Glücksstrebens gebührt. Liberale Demokratien können sich jedoch nicht darauf beschränken, negative Freiheitsrechte zu garantieren, sondern müssen überdies sicherstellen, dass alle Bürgerinnen und Bürger die ihnen versprochenen Rechte tatsächlich ausüben können und zu diesem Zweck, also nicht zum Zweck eines glücklichen Lebens, über einen angemessenen Anteil an bestimmten Gütern verfügen müssen. Gerechte Politik hat somit nicht nur Freiheiten gleich zu verteilen, sondern nach Möglichkeit dafür zu sorgen, dass der Wert der Freiheiten für alle gleich groß ist, dass also auch jene, die nicht über Macht und Reichtum verfügen, im Rahmen der ihnen zustehenden Freiheitsräume ihre Ziele erreichen können. Auch hat sie den fairen Wert der politischen Freiheit zu sichern, indem sie es allen ermöglicht, politisch zu partizipieren, und zu diesem $Z$ weck die Konzentration ökonomischer Macht verhindert (vgl. Rawls, 1979, S. 232 f, 255 ff).

Der egalitär-liberale Begriff distributiver Gerechtigkeit unterscheidet sich sowohl vom aristotelischen wie vom Kant'schen Begriff. Die Herstellung von Gerechtigkeit erweist sich bei Rawls von Anfang an als ein Problem distributiver Politik. Gerechtigkeitsprinzipien sind auf die Grundstruktur der Gesellschaft anzuwenden; dabei handelt es sich um einen Verteilungsmechanismus, der ungleiche Lebenschancen bewirkt. Die Korrektur erfolgt durch die Neuverteilung derjenigen sozialen Güter, die für die Befriedigung allgemein menschlicher Bedürfnisse und die Ausführung vernünftiger Lebenspläne nötig sind (Rawls, 1979, S. $112 \mathrm{ff}$ ); dazu gehören politische Rechte und bürgerliche Freiheiten, Machtpositionen, Chancen und materielle Güter. Offenbar kann bei der Bestimmung der Grundrechte die natürliche und soziale Bedürftigkeit des Menschen nicht außer Acht gelassen werden. Egalitär-liberale Theorien postulieren zusätzlich zum Recht auf Autonomie ein Recht auf die Bedingungen, die autonomes Handeln ermöglichen; solche Bedingungen reichen von der Grundversorgung über die Bildung bis zum Einbezug in ein politisches Gemeinwesen. Henry Shue postuliert „basic rights“, die den Machtlosen als Verteidigungsmittel zustehen sollten. Basale Rechte umfassen die Rechte auf Sicherheit und Subsistenz sowie politisch-gesellschaftliche Partizipationsrechte. Ein Recht ist dann basal, wenn seine Ausübung notwendige Bedingung für die Ausübung jedes anderen möglichen Rechts ist (Shue, 1996, S. 18 f; vgl. Jones, 1999, S. 50-84).

Die „Positivierung“ liberaler Freiheitskonzeptionen ist angesichts der über Gewaltanwendung hinausgehenden, ökonomische, soziale und Kommunikationsmacht einbeziehenden Vielfalt möglicher Gefährdungen individueller Selbstbestimmung logisch. Der Staat kann die gleiche Freiheit nicht ausschließlich formalrechtlich garantieren, er muss gesellschaftliche Macht kontrollieren und begrenzen, die sozialen Voraussetzungen zur Freiheitsrealisierung sichern und darauf hinwirken, dass die individuelle Realisierung der Freiheit allen im gleichen Maß möglich ist (vgl. Böckenförde, 1991, S. 264-276). Die Positivierung führt aber dazu, dass Legitimitätskriterien politischer Ordnung anspruchsvoller werden und ihre Definition komplizierter wird. Für Kant reduziert sich der distributive Akt auf die Einteilung jener Räume, worin das Recht jedes Individuums auf gleiche Handlungsfreiheit garantiert ist. Bedingung gleicher Freiheit ist in egalitär-liberaler Sicht indes eine gesellschaftliche Ordnung, die mittels allgemeiner Gesetze die 
Freiheit aller einschränkt und zugleich eine gerechte Verteilung jener sozialen Güter garantiert, die für ein selbstbestimmtes Lebens erforderlich sind (Koller, 1998b, S. 495). Prinzipien distributiver Gerechtigkeit sind laut Peter Koller dort zu bestimmen, wo „die gesellschaftliche Ordnung Güter und Lasten in Gestalt von Rechten und Pflichten, Freiheiten, Machtbefugnissen, sozialen Positionen und Lebenschancen verteilt“ (Koller, 1994, S. 129). Rechtliche Gleichheit, bürgerliche Freiheit und demokratische Beteiligung resultieren in dieser Sichtweise ebenso aus gerechter Distribution wie soziale Chancengleichheit und wirtschaftliche Verteilungsgerechtigkeit. Koller glaubt überdies, ein annehmbares Verfahren zur Feststellung der Vereinbarkeit gleicher Freiheit, das nicht zugleich die Folgen des allseitigen Freiheitsgebrauchs im Hinblick auf die Zwecke und Interessen der Menschheit bewertet, sei kaum zu finden. Eine solche Bewertung setzt eine bestimmte Vorstellung des Guten voraus; somit ist es nicht möglich, das Rechte unabhängig von einer Konzeption des Guten zu bestimmen (Koller, 1994, S. 146 f).

Denkbar wäre beispielsweise, dass im Interesse der Überlebens-, Entwicklungs- und Selbstbestimmungschancen der Mehrheit der Weltbevölkerung das Recht der Minderheit, ihr Konsumverhalten frei zu wählen, negiert würde. Anders formuliert: der Anspruch aller Erdbewohnerinnen und -bewohner auf ein sicheres und selbstbestimmtes Leben verlangt von einigen, ihren Lebensstil im Sinne der Einschränkung des Konsumverhaltens auf ein allen zuträgliches Maß zu verändern. Wahlfreiheit stößt dort auf Grenzen, wo die gewählte Konzeption des Guten, sei es eine intolerante Religion, sei es eine bestimmte Art des Ressourcenverbrauchs, unzumutbare Auswirkungen auf die Freiheit der anderen hat; das Prinzip des Vorrangs der gleichen Freiheit wird dadurch nicht in Frage gestellt. Die Frage, wann von gleicher Freiheit, gleichen Handlungsmöglichkeiten, gleichen Voraussetzungen für ein selbstbestimmtes Leben tatsächlich die Rede sein kann, wird jedoch unvermeidlich stets neu zum Gegenstand politischer Auseinandersetzungen.

Besonderes Gewicht kommt der Frage, ob eine gerechte politische Ordnung bei der Bestimmung des Umfangs und der Grenzen individueller Freiheit auf eine Konzeption des guten Lebens rekurrieren muss, in einem namentlich von Martha Nussbaum und Amartya Sen vertretenen linksliberalen Aristotelismus zu. Sen unterscheidet die Primärgüter, die nach Rawls gleich zu verteilen sind, als Mittel zum Zweck der Freiheit vom Zweck selbst, dem Umfang der Freiheit (Sen, 1992, S. XI). Freiheit ist dann allerdings, so müsste der klassisch-liberale Einwand lauten, gleichbedeutend mit dem guten Leben, die Vorrangregel somit hinfällig. Nussbaum definiert staatliche Aufgaben explizit im Sinnne der Priorität des Guten. Der von ihr vertretene sozialdemokratische Aristotelismus schreibt der Politik eine umfassende Kompetenz zu, soll doch ihre Sorge allen wichtigen Tätigkeiten jeder einzelnen Bürgerin und jedes einzelnen Bürgers gelten. Freilich darf, so präzisiert Nussbaum, aristotelische Politik nicht in paternalistischer Manier den Menschen eine bestimmte Konzeption des Guten aufzwingen, vielmehr ist sie zuständig für die Bereitstellung der materiellen und institutionellen Voraussetzungen, die die Menschen zu bestimmten Tätigkeiten befähigen und ihre autonome Entscheidungsfähigkeit fördern. Gerechte Politik ist in dieser Sichtweise zwar notwendig 
distributive Politik, zielt aber nicht primär auf materielle Umverteilung, sondern auf die Beseitigung aller Hindernisse, die zwischen Menschen und der vollen Entfaltung ihrer Fähigkeiten stehen, wozu inbesondere asymmetrische Machtbeziehungen gehören, wie sie aus dem Privateigentum an Produktionsmitteln oder aus patriarchalen Strukturen resultieren (Nussbaum, 1999, S. 40-43).

Die Infragestellung der klassisch-liberalen Vorrangregel im linksliberalen Aristotelismus bedeutet keine Relativierung des Prinzips individueller Freiheit, vielmehr resultiert sie aus einem radikalen Verständnis positiver Freiheit. Sens Begriff positiver Freiheit ${ }^{5}$ bezeichnet die Fähigkeit zu handeln, in einem elementaren Sinn die Möglichkeit, irgendetwas zu sein und zu tun. Er meint im Besonderen Wahlfreiheit, die Fähigkeit, sich zwischen verschiedenen Alternativen zu entscheiden, und die Macht, gemäß der getroffenen Entscheidung zu handeln. Selbstverständlich setzt so verstandene Freiheit die Abwesenheit von Zwang voraus, doch versteht Sen, anders als Berlin (vgl. Berlin, 1995, S. 201-211), aber ähnlich wie Marx, Unfreiheit auch als Ergebnis fehlender Handlungs- und Entfaltungsmöglichkeiten, die durch soziale Verhältnisse bedingt sind, als strukturellen Zwang. Handlungsfreiheit setzt die Befriedigung von Bedürfnissen, die Entwicklung von Anlagen voraus; die politische Philosophie muss mithin ihre Aufmerksamkeit auf menschliche Funktionsweisen (functionings) und Fähigkeiten (capabilities) lenken (vgl. Nussbaum/Glover, 1995, S. 72-86).

Auf die Frage nach den Bedingungen der Möglichkeit, frei zu handeln, gibt auch Onora O'Neill, die von Kant geprägt ist, eine neue und radikale Antwort. Philosophische Theorien, die die Erfahrungen von Ohnmacht und Fremdbestimmung ausblenden, wie sie die Situation eines großen Teils der Weltbevölkerung prägen, können den Anforderungen nicht genügen, die an eine den globalen Verhältnissen angemessene Konzeption von Gerechtigkeit zu stellen sind, so ihr Argument. Solche Theorien scheitern allerdings nicht aufgrund ihrer notwendig abstrakten Begründungsmodelle, sondern gerade deswegen, weil ihre Abstraktionen unvollständig sind, weil sie die Handlungsmöglichkeiten privilegierter individueller und institutioneller Akteure idealisieren und unterstellen, alle Akteure verfügten über diese Möglichkeiten. Gerechtigkeitstheorien sind folglich aufgrund ihres speziellen Sensoriums für die effektiven Bedingungen der Möglichkeit des Entscheidens und Handelns zu beurteilen. Die Gerechtigkeit von Institutionen und Prozeduren ist daran zu messen, ob diese es Menschen selbst in Situationen politischer Ausgrenzung und ökonomischer Abhängigkeit, insbesondere armen Frauen in armen Ländern, erlauben, die ihnen zugewiesenen Positionen zu verweigern oder neu auszuhandeln (O’Neill, 2000, S. 143-167).

Die Verbindung der aristotelischen Ethik der Selbstverwirklichung mit dem Autonomieideal der Aufklärung bildet das normative Fundament der Philosophie von Marx, und der aristotelische Linksliberalismus der Gegenwart ist mit Problemen konfrontiert, die bereits Marx' Versuch, eine Konzeption positiver Freiheit als oberstes Legitimitätskriterium politischer Ordnung zu bestimmen, begleiten. Je

5 Zu Sens Freiheitsverständnis besonders Sen 1990, S. 116; 1992, S. 31-34; Crocker, 1995, S. $182 \mathrm{ff}$. 
besser es der Philosophie gelingt, konkrete Voraussetzungen menschlicher Handlungsfreiheit in den Blick zu bekommen, desto schwerer fällt es ihr, den gerechtigkeitsrelevanten Gegenstand der Gleichverteilung zu bestimmen (vgl. Cohen, 1993) und derart die konkrete Aufgabe distributiver Politik zu benennen. Sen fragt in diesem Kontext nach der „Fokalvariablen“ (Sen, 1992, S. 2); die Frage ist für ihn auch deshalb entscheidend, weil gerade eine egalitäre Gerechtigkeitskonzeption vom Faktum der Ungleichheit der Menschen ausgehen muss. Diese Einsicht teilt Sen mit Marx, der seine sozialdemokratischen Zeitgenossen mit einer akribischen Erörterung der Frage nach der gerechten Verteilung verwirrt hat. Weil Menschen hinsichtlich ihrer Fähigkeiten und Bedürfnisse ungleich sind, ist gleiches Recht im Effekt immer „ein Recht der Ungleichheit“. Marx kritisiert das sozialistische Programm, weil es auf die Verteilung von Konsumtionsmitteln zielt und nicht auf die Verteilung der Produktionsbedingungen (Marx, MEW 19, S. 18-22). Erst die breite Verteilung technischen und praktischen Wissens ermöglicht den Abbau asymmetrischer Machtverhältnisse.

Sen und Nussbaum haben der Frage von Marx neues Gewicht gegeben: Wie sind egalitäre Kriterien zu bestimmen, damit sie in der Anwendung nicht ein „Recht der Ungleichheit“ sanktionieren. Jenes Gut, von dem allen Menschen ein gleicher Anteil zusteht, wäre als positive Freiheit oder Handlungsvermögen zu bezeichnen. Seine gerechte Verteilung ist aus mehreren Gründen ein heikles Projekt. Der Bedarf danach differiert zwischen Individuen, Geschlechtern, Klassen und Nationen stark und ist schwer zu bemessen. Überdies sind „substantive freedoms“ (Sen, 1999, S. 13-86) auf zahlreiche Voraussetzungen wie Nahrung und Gesundheit, Bildung, politische Partizipation und das Fehlen ökonomischen Zwangs angewiesen. Kann ein Recht auf all diese Voraussetzungen postuliert werden? Marx hat seine Theorie bekanntlich nicht in der Sprache des Rechts formuliert. Seine Kritik der liberalen und utilitaristischen Freiheitsauffassung ist jedoch aufschlussreich und zeigt mögliche Elemente einer radikalen Rechtskonzeption. In der Zirkulationssphäre erkennt er das Reich der angeborenen Menschenrechte, worin alle Menschen frei und gleich sind, Eigentum besitzen und ihren Eigennutz verfolgen (Marx, MEW 23, S. 189f). In der Produktionssphäre dagegen, so die unausgesprochene Pointe des Arguments, findet kein freiwilliger und für beide Seiten vorteilhafter Tausch statt. Die Verkäufer der Ware Arbeitskraft sind weitgehend rechtlos, sie stehen den Käufern nicht als gleichberechtigte Vertragspartner gegenüber, da der Mangel an Subsistenz- oder Produktionsmitteln sie zum Abschluss eines für sie unvorteilhaften Arbeitsvertrags zwingt. Im Rahmen einer radikalen Philosophie wäre also ein Recht nicht bloß als ein Anspruch zu verstehen, vor der Gewalt anderer Menschen beschützt zu werden, sondern als Instrument der Abwehr jeder Art von Fremdbestimmung und Machtausübung. Distributive Gerechtigkeit würde neben diesem negativen auch einen aktiven und einen positiven Aspekt umfassen: den Anspruch auf Partizipation an der Gestaltung nicht bloß der politischrechtlichen, sondern auch der sozialen und ökonomischen Verhältnisse sowie den Anspruch auf individuelle Selbstverwirklichung und die dazu nötigen wissenschaftlichen und kulturellen Ressourcen. Materielle Umverteilung ist dabei ein Mittel zum Zweck. 


\section{Offene Fragen}

Die Einsicht wächst, dass eine auf das Wachstum des Bruttosozialprodukts fixierte Entwicklungspolitik scheitern muss, weil sie die Ursachen von Armut und Abhängigkeit nicht zur Kenntnis nimmt. Monetäre Indikatoren können die Realität der Armut nur ungenügend erfassen, obgleich sie in einer stark monetarisierten Wirtschaft nicht zu vernachlässigen sind. Armut bedeutet in erster Linie Ohnmacht und Fremdbestimmung, die Einschränkung des eigenen Handlungspielraums und fehlende Einflussmöglichkeiten. Philosophie wie Politik scheinen zu zögern, die Konsequenzen aus solchen Einsichten zu ziehen.

Die Widerstände der Philosophie sind nachvollziehbar. Selbst wenn zugestanden wird, dass die Aufgabe distributiver Politik in der gleichen Verteilung von Freiheit, Handlungsmacht und Entwicklungschancen besteht, bleibt offen, was konkret zu verteilen ist. Die Faktoren, die Menschen in ihrer Handlungsfähigkeit einschränken, können beträchtlich differieren. Spezielle Bedürfnisse bilden sich aufgrund der jeweiligen Position im Netz sozialer Abhängigkeiten heraus. Daher müssen Maßnahmen, die Abhilfe schaffen können, stets neu bestimmt und geprüft werden. Nötig sind zu diesem Zweck offene Informationsflüsse und funktionierende Partizipationsmechanismen. Die individuellen Chancen, Fähigkeiten entwickeln und einsetzen zu können, können auf nationaler wie globaler Ebene letztlich nur dann besser verteilt werden, wenn Machtungleichgewichte korrigiert werden.

Weil die Ohnmächtigen und Benachteiligten unter ganz unterschiedlichen Formen von Entbehrung, Unterdrückung und fehlender Wahlfreiheit leiden, können nur sie selbst bestimmen, welche Art der Neuverteilung ihre Situation nachhaltig verbessern kann. Soll Umverteilung dem Zweck der generellen Verbesserung der Lebensbedingungen dienen, setzt sie Kommunikationsformen voraus, worin Erfahrungen schlechter Lebensbedingungen sich artikulieren können, sowie eine möglichst breite und gleichberechtigte Beteiligung an Entscheidungsprozessen. Globale distributive Gerechtigkeit erfordert daher zunächst einmal die Institutionalisierung übernationaler demokratischer Verfahren, wozu transparente Verhandlungspraktiken und ein symmetrischer Austausch von Wissen gehören. In künftigen Institutionen müssen, wenn sie denn ihr Ziel, die gerechtere Verteilung von Freiheit, erreichen sollen, gerade jene Menschen einen größeren Anteil an der Entscheidungsmacht erhalten, die am stärksten unter Ohnmacht und Fremdbestimmung leiden: nicht nur die Regierungen armer Nationen, nicht nur die in NGO’s organisierte Zivilgesellschaft, sondern Vertreter jener Bevölkerungsgruppen, die von externen Effekten der globalen Marktliberalisierung am direktesten betroffen sind, Gewerkschaften, Frauenorganisationen, Repräsentanten jener Frauen und Männer, die in der Geschlechterhierarchie und in hierarchischen Arbeits- und Produktionsverhältnissen auf den untersten Stufen stehen und deren Stimmen bislang kaum Gehör gefunden haben.

Die Widerstände der Politik erklären sich aus ihrer freiwilligen oder unfreiwilligen Anpassung an die Sachzwänge des globalen Kapitalismus. Dieser hat Institutionen geschaffen, die vor allem im finanz- und handelspolitischen Bereich die Welt zu einer Kooperationsgemeinschaft zusammenschweißen, worin Nutzen 
und Lasten höchst ungleich verteilt sind. Der Westen hat die Agenda einer Globalisierung bestimmt, die die Armut nicht verringert, die Stabilität nicht erhöht, wovon er aber auf Kosten der Entwicklungsländer überproportional profitiert (Stiglitz, 2002, S. 20 ff). Die vielen Ländern aufgezwungene Liberalisierung der Kapitalmärkte hat oft wenigen Anlegern genützt, unzählige Arbeitsplätze vernichtet und ganze Volkswirtschaften in den Ruin getrieben. Agrarsubventionen in reichen Ländern können die Lebensgrundlagen zahlloser Menschen in armen Ländern zerstören. Billige Arbeitskraft und Arbeitsbedingungen, die ILO-Standards nicht genügen, können als „komparative Vorteile“ hohe Profite und tiefe Warenpreise bewirken, ohne dass ein marktwirtschaftlich orientiertes Denken die Frage, wie die Leidtragenden kompensiert werden können, ernsthaft stellen würde. Ökologische Folgen des Verbrauchs bestimmter Ressourcen in reichen und armen Ländern können sich für arme Länder verheerend auswirken. Die Globalisierung von Produktion, Märkten und Konsum schafft vielfältige Interdependenzen, und die politische Philosophie muss prüfen, wo gerechtigkeitsrelevante Beziehungen zwischen Ursache und Wirkung vorliegen. Globale distributive Gerechtigkeit kann allerdings nur im Zuge der Infragestellung des paternalistischen Kompetenzanspruchs sowie der monopolartigen Entscheidungs- und Sanktionsmacht der internationalen finanz- und handelspolitischen Institutionen gefördert werden. Der Weg dazu muss über stärkere Marktregulierungen führen, über besseren Schutz vor Marktexternalitäten und das Recht auf demokratische Entscheidungen in der Wirtschaftspolitik. Er kann nur beschritten werden, wenn die Emanzipation von neoliberalen Dogmen gelingt. Der vielfach widerlegte „Washington Consensus“, der Hilfe suchenden Ländern Marktöffnung, Privatisierung und fiskalische Einschränkung empfiehlt, zeigt exemplarisch, wie eine wissenschaftlich fragwürdige und gerechtigkeitsblinde Dogmengläubigkeit das bestehende Macht- und Wohlstandsgefälle zementiert.

Globale distributive Gerechtigkeit verlangt nicht primär die Umverteilung materieller Güter, sondern die Regulation unkontrollierter Mechanismen, die wachsende Fremdbestimmung und negative Umverteilungseffekte zur Folge haben. Solche Mechanismen dienen häufig mächtigen Partikularinteressen, die in internationalen Institutionen über überproportionales Gewicht verfügen. Die gerechtere Gestaltung der Welt wird ohne einschneidende ökonomische Regulierungen und eine Neudefinition der Verfügungsmacht über die Produktionsmittel kaum möglich sein, mithin auf starken Widerstand der Nutznießer der gegenwärtigen Verteilungsordnung stoßen. Wiederum wird klar, dass deren Veränderung nur möglich ist im Rahmen einer globalen Demokratisierung, eines individuellen und kollektiven „empowerment“ der bislang Machtlosen. 